\author{
Military Technical College \\ Kobry El-Kobbah, \\ Cairo, Egypt.
}

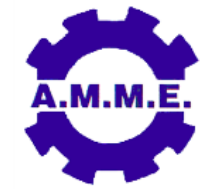

$17^{\text {th }}$ International Conference on Applied Mechanics and Mechanical Engineering.

\title{
FORMATION OF SUPER SATURATED SOLID SOLUTION FE-50WT.\%CU BY HIGH ENERGY BALL MILLING (ATTRITOR)
}

\author{
A. E. Hannora*, F. F. Hanna and L. K. Marei
}

\begin{abstract}
High energy ball milling (attritor) was performed to synthesize Cu-50wt.\% Fe super saturated solid solution at room temperature. The mechanically alloyed samples have been milled for $24 \mathrm{hrs}$ and examined by X-ray diffraction (XRD) to investigate its microstructure. As a result of mechanical deformation a higher defect concentration generated in the powder and thus the crystallinity decreases as well as crystallite size. The magnetic properties assessments of as-milled samples were carried out using vibrating sample magnetometer (VSM). The magnetization decreases with milling time while the corecivity increases up to $12 \mathrm{hrs}$ milling and then decreases.
\end{abstract}

\section{KEY WARDS}

Fe-Cu, Mechanical milling, magnetic properties

Suez University, Faculty of Petroleum and Mining Engineering, Science and Mathematics Department, Suez, Egypt, 43721.

* Corresponding author's, Tel. +201147043930, Fax: +20623360252, E-Mail: ahmed.hannora@suezuniv.edu.eg 


\section{INTRODUCTION}

The Fe-Cu binary system is known to have low solid miscibility, and solubility is limited to only $3 \%$. Uenishi et. al. [1] and Yavari et. al. [2] were the first to report the formation of metastable solid solutions in the $\mathrm{Fe}-\mathrm{Cu}$ system using MA technique. A wider range as well equiatomic composition have been explore and there phases formation and stability reported is depend to large extent on the different processing techniques used to produce the alloys. The majority of research concentrated on the formation of metastable phases sings to phase of $\mathrm{Cu}-\mathrm{Fe}$ produced from the elemental powder blends with a positive heat of mixing. In spite the extensive studies on the system, there are many disagreement on the literature concerning the formation of single phase solid solution with homogeneous structure is possible or not $[3,4]$

The magnetism of iron is related to its crystal structure, and it is well known, that $\mathrm{Fe}$ is ferromagnetic up to $1043 \mathrm{~K}$ when the conventional body centered cubic (bcc) structure is maintained, while $\mathrm{Fe}$ in face centered cubic (fcc) structure, stable at high temperature (above $1183 \mathrm{~K}$ ), is non-magnetic [5]. Moreover, the stabilization of Fe fcc structure, the so called $\mathrm{Y}-\mathrm{Fe}$, at room temperature (RT), can be achieved by substituting $\mathrm{Fe}$ atoms in a simple fcc lattice, such as fcc-Fe-TM those of some pure metals $(\mathrm{Ni}, \mathrm{Cu}, \mathrm{Pt}, \mathrm{Pd})$. When one of the immiscible $\mathrm{Fe}$ is ferromagnetic, the decomposition, by mechanical alloying (MA), of the supersaturated phases can allow development of low-cost new magnetic materials with large magnetoresistance and coercivities, unusually strong magnetocaloric effects, and high thermal/electrical conductivities usually unattainable in permanent magnet [6].

The aim of this work was to study the structural transformation taking place during mechanical alloying of $\mathrm{Cu}_{50} \mathrm{~F}_{50}$ mixture in a high energy ball mill. The produced structure of the milling were evaluated using XRD as well as scan electron microscopy. The type of the magnetic properties of the new materials was also examined.

\section{MATERIALS AND SAMPLES}

Iron 99\% and copper 99.9\% -325 mesh powders (Alfa Asar) were placed into high energy ball milling modified attritor, rotating with $500 \mathrm{rpm}$. The powders were treated with hard steel balls at different milling times with the optimum balls to powder ratio equals to $10: 1$.

XRD was performed using D5000 powder diffractometer using $\mathrm{Cu} \mathrm{K}_{\alpha}$ radiation (wavelength $\lambda=0.15406 \mathrm{~nm}$ ) with a nickel filter at $40 \mathrm{kV}$ and $30 \mathrm{~mA}$. The diffractometer was operated within range of $10^{\circ}<2 \theta<100^{\circ}$ with one second step-time and 0.05 degree step-size. Diffraction signal intensity throughout the scan was monitored and processed with DIFFRAC plus software. Topas 2.1 Profile fit software was used to extract the peak parameters and the Pseudo Voigt function was used to model the peak shape. The XRD patterns of the synthesized samples were compared with ICDD (JCPDS) standard cards, Iron (01-1267) cubic phase with space group Im3m (229) and copper (85-1326) hexagonal phase with space group Fm3m (225). The magnetic properties of the specimen were measured by a Vibrating Sample Magnetometer Lake Shore model 7410 (USA). 


\section{RESULTS AND DISCUSSION}

\section{X-Ray Diffraction}

Figure (1) presents the X-ray diffraction patterns of ball milled Cu-50wt.\%Fe powder mixture. The mixture was milled for different periods up to $24 \mathrm{hrs}$. The XRD pattern of the elemental powder mixture before MM/MA shows only sharp peaks corresponding to the both metal mixture. The $\mathrm{Fe}_{50} \mathrm{Cu}_{50}$ samples set as a function of milling time are shown in Fig. (1). A notable intensity reductions occur as a result of severs plastic deformation after two hours milling. No additional possible new phases were detected throughout the MA process up to $7 \mathrm{hrs}$. The peaks of Fe phase essentially disappear after $17 \mathrm{hrs}$ milling while very broad peaks closed to (111) Cu phase developed with milling time. This implies that $\mathrm{Cu}$ has the lowest alloying rate among the mechanically milled elements. Moreover, disappearing of the Fe diffraction lines can be taken as indication that iron is integrated in the fcc-Cu structure and possible given rise to the formation of solid solution phase leading to diffraction peaks shift to higher angles progressively with milling time with increasing Fe-solubility in $\mathrm{Cu}$. Following mechanical milling for $12 \mathrm{hrs}$, the main peak (110) intensities of bcc Fe-phase is enhanced while fcc Cu-phase main peak (111) decreases. Such an increase of (110) - Fe peaks started after $7 \mathrm{hrs}$ milling could be attributed to the relative high dislocation density reach a saturation value, and the existing dislocation rearrange and some may be annihilated. Therefore, with further milling, the lattice strain on iron peak decreases and become sharper and even the peak position may change in reverse order. On the other hand, the (111) peak continuous to further broadening and reduced in intensities which indicate a higher work hardening rate for copper compare to the second mixed element iron. Therefore, copper continuous deformed with milling with subsequent increases in lattice strain and rather the newly formed solid solution particle refinement. A 24 hours milling is needed for the powder finally transforms into very fine structure and or amorphous as a result of the accumulated energy stored produced by milling.

The relative crystallinity was roughly calculated by comparing the integrated area intensity under peaks, of the MA powder with the as-received powders as a reference. From the results of the profile fitting, the relative crystallinity of the fcc Cu-phase was sharply decreased after $2 \mathrm{hrs}$ of MA. The bcc Fe-phase shows relative crystallinity enhancement after $7 \mathrm{hrs}$ of MA and then decreases to the minimum value after $17 \mathrm{hrs}$.

The analysis of the produced phases after different stages of milling the $\mathrm{Cu}-\mathrm{Fe}$ elements powder can be in general divided into two parts.

\section{The Formation of Nanocrystalline Supersaturated Structure}

The elements $\mathrm{Fe}$ and $\mathrm{Cu}$ are known to be immiscible and there is no intermediate phase exist on the equilibrium diagram. Moreover, the $\mathrm{Cu}$ elements are unique case where they differ only by $1.5 \%$ in atomic radius and they are very close in electron negativity, $1.9 \mathrm{Cu}$ and $1.8 \mathrm{Fe}$, with a solubility limit near $0.3 \%$ at room temperature. To monitor the alloying process after the various stages of milling, The XRD results are examined and used to calculate the change of the d-spacing values as shown in Fig. (2). 


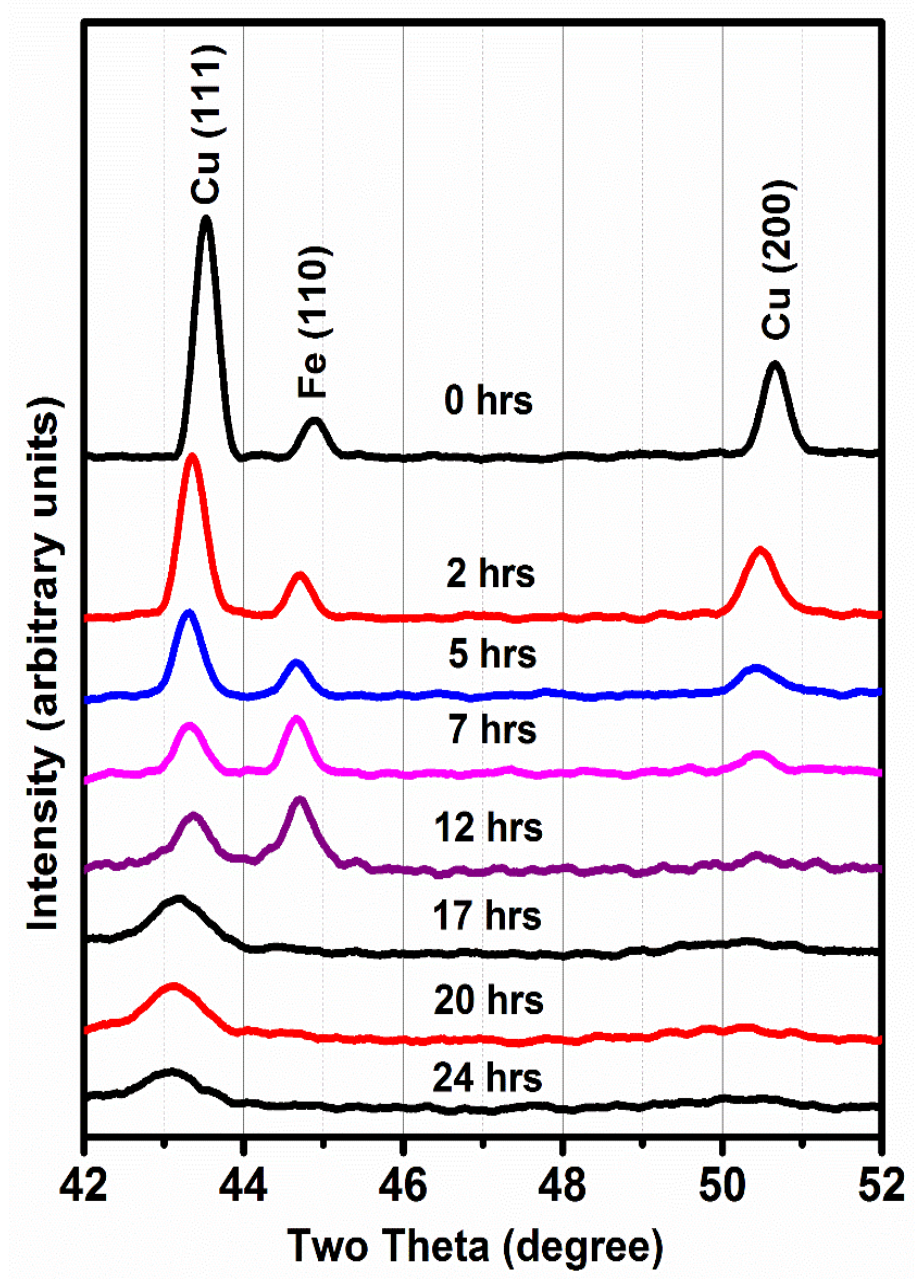

Fig. 1. X-ray diffraction patterns of mechanically milled Cu-50wt.\%Fe powder at different milling time.

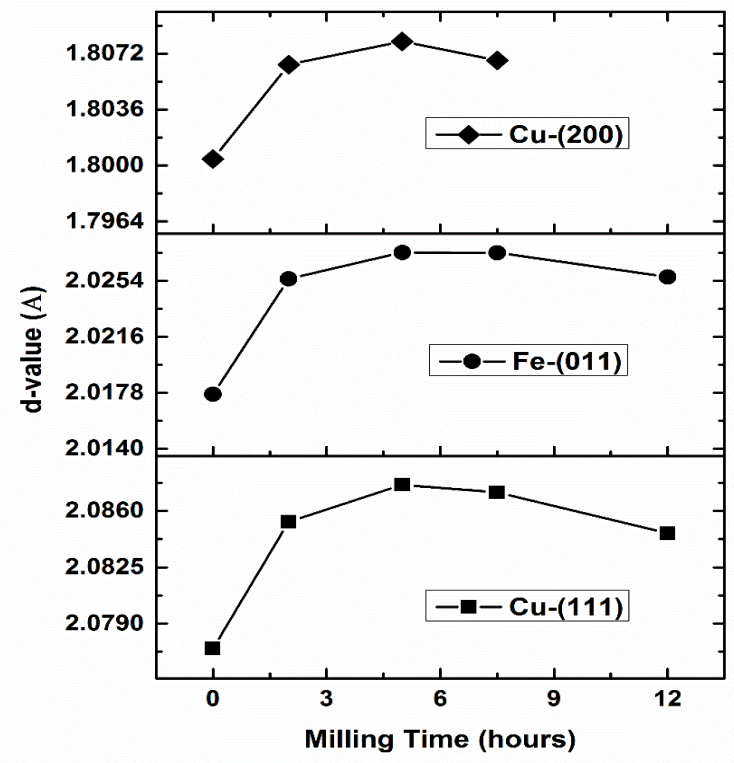

Fig. 2. D-values variation after mechanical alloying with different time. 
The results indicate that the peaks intensities reduction are associated with shift in peaks position as milling time increase and lead to a subsequent reduction of particle size of both elements, $\mathrm{Fe}$ and $\mathrm{Cu}$. This stage of milling continuous up to $12 \mathrm{hrs}$ and the observed changes could be related mainly to the increase of heterogeneous strain on the lattice of both $\mathrm{Fe}$ and $\mathrm{Cu}$ as a result of high milling energy. The continuous changes in peak profiles, broadening and displacement, are a demonstration that Fe have gradually react with $\mathrm{Cu}$ to form a super-saturated $\mathrm{Cu}(\mathrm{Fe})$ limited solid solution phase. The present result suggested that the fcc on lattice is expanded, with milling time up to $12 \mathrm{hrs}$, by incorporation of iron atom and resulted in an increase of the lattice parameter from $0.3615 \mathrm{~nm}$ to $0.3451 \mathrm{~nm}$ for $\mathrm{Cu}(\mathrm{Fe})$ solid solution. This result is in agreement with previously obtained values [7,8], in spite the fact that iron-atomic size is slightly larger by regard to copper. It is therefore, possible to conclude that at end of this milling stage $17 \mathrm{hrs}$, a mixture of an $\mathrm{Cu}(\mathrm{Fe})$ supersaturated phase is formed in addition to fcc $(\mathrm{Cu})$ and both have a nanometer particle size.

Mössboure spectroscopy analysis is also used by several authors to examine the possible local atomic alloying after milling CuFe mixtures [1, 9, and 10]. In general their results indicated that after the stages of milling up to $20 \mathrm{hrs}$, two nonmagnetic spectra are observed and attributed to the $\mathrm{Fe}(\mathrm{Cu})$ solid solution and a fcc $(\mathrm{Fe})$ phase attached to the fcc $(\mathrm{Cu})$ matrix. The results confirm the presence of more than one phase, but it differ in suggesting a transformation of bcc (Fe) rich in copper to an fcc (Fe) structure take place during this stage of milling. Moreover, it is important to indicate that a maximum of 3at.\%Cu concentration is estimated in fcc (Fe) phase. Such transit phase is not detected up to $17 \mathrm{hrs}$ of milling, where all possible Fe-peak completely disappears which could be due to the formation of a small amount of such bcc (Fe) phase in addition to the expected broadening due to lattice strain. Under such condition it would rather difficult to identify the presence of such phase. Malt et al [11] indicated that based on bond measurement between $\mathrm{Cu}$ and $\mathrm{Fe}$ that neither a very weak interaction nor repulsion exist between unlike neighbor of $\mathrm{Cu}$ and $\mathrm{Fe}$ atoms and been attributed to the positive heat of mixing in this system.

\section{Formation of (Cu-Fe) Solid Solution Phase.}

Figure (1), shows that further milling time between 17 to $24 \mathrm{hrs,} \mathrm{only} \mathrm{a} \mathrm{single} \mathrm{peak}$ broad and relatively strong in intensity can be observed. This peak is broad enough to include all the remaining of (110)-Fe peak. At this stage of high milling time, further refinement of the microstructure is expected due to the increase in defect density as well as an increase of interface area of particles. Under such condition in the diffusion between $\mathrm{Cu}$ and $\mathrm{Fe}$ will be more favorable and could result in alloying between elements particles on the atomic level. Simulation calculation results suggested that the formation of solid solution is possible for a system with positive heats of mixing at a relative moderate shearing rate [12].

There is some disagreement in the literature over the occurrence of a complete atomic level solid solution in the processing CuFe powder by mechanical alloying. The confliction could be mainly associated with difficulties in analysis XRD results; the existing of high defect crystallites with a few nanosize contributing to further broadening, which make it hard to identify the peaks. In that respect, scan electron microscopy image has been used for microstructure clarification. Figure (3) show example for FeCu 
particle after $7 \mathrm{hrs}$ milling, where an almost homogeneous particles distribution can observed with a nanonsize particle size conforming the XRD results. At a higher magnification, it is possible to observe the flat layer nature of the particles as formed by milling, a very fine particles in the order of a few micro scan can be also observed inside the larger particles mainly at the center. This microstructure observation can indicate that due the continuous increased deformation during milling harder $\mathrm{Fe}$ compare to Cu particles will be fragment to very small particles as the one observed in the scan micro graph. This arrangement has been indicated by both Yavari et al [2] and Genete et al [13], to be much favorable site for atoms to be continuous dissolve under deformation. They further estimated the enthalpy associated with generated very fine dissolve particles and they concluded that for such small size particles the formation of solid solution can energetically possible. However, there are a disagreement on the nature of the interface between element component formed after dissolution, it either coherent or incoherent.

A more recent study using high resolution TEM, confirmed the formation of dual phase microstructure rather than a single $\mathrm{Cu}(\mathrm{Fe})$ solid solution. A clusters of atoms are found to be distributed randomly in the $\mathrm{Cu}(\mathrm{Fe})$ solid solution matrix [3]. Therefore, it is possible to conclude the $\mathrm{Fe}(\mathrm{Cu})$ particle formed inside the supersaturated $\mathrm{Cu}$-rich in Fe solid solution after milling in the high energy ball-mill after $24 \mathrm{hrs}$.
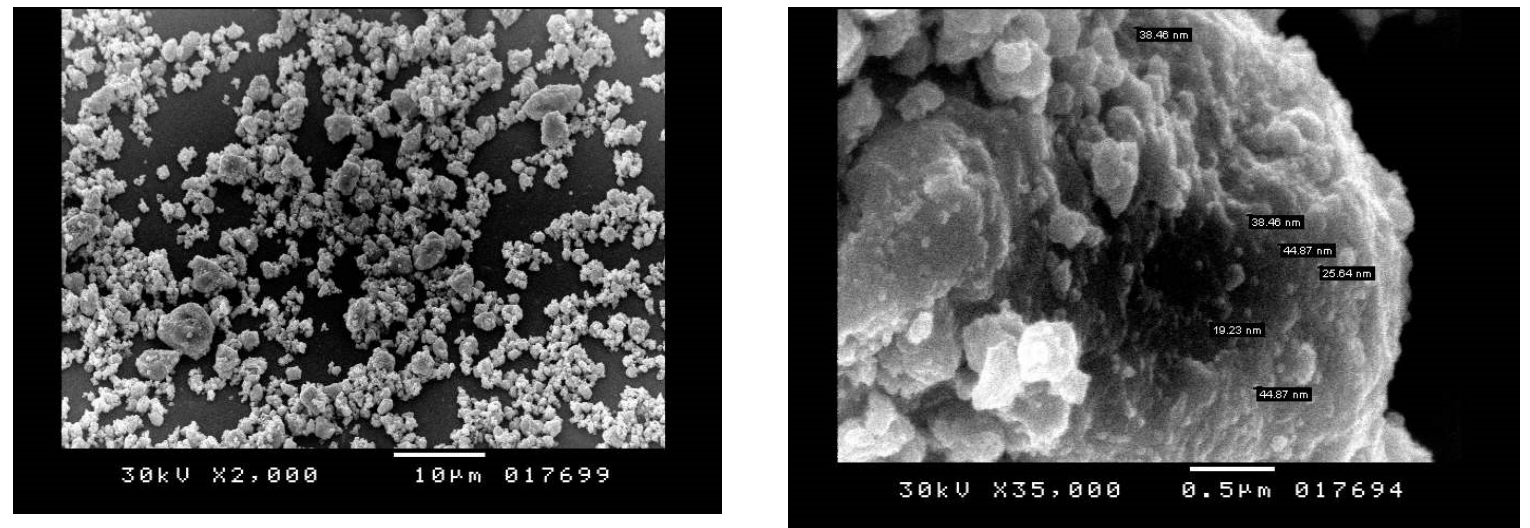

Fig. 3. SEM images of mechanical alloyedFe-50wt.\%Cu for $7 \mathrm{hrs}$.

Figure (4) shows the VSM results of the Fe ${ }_{50} \mathrm{Cu}_{50}$ milled samples after different milling times, which consists of ferromagnetic iron in $\mathrm{Cu}$ matrix at short time of mechanical alloying. A super-paramagnetic behavior was observed after higher time of milling and could be related to the fine structure formation (very small domain).

Figure $(5 \mathrm{a})$ presents the variations of both the coercivity and magnetization at room temperature of $\mathrm{Fe}-50 \mathrm{wt} . \% \mathrm{Cu}$ powder with milling time. The coercivity and magnetization values at 0,2 , and $5 \mathrm{hrs}$ milling time can be attributed to the solid solution refinement stage. Following further milling between 5 to $12 \mathrm{hrs}$, a relative higher rate of coercivity increases are obtained, from $\sim 20$ to $\sim 105 \mathrm{G}$ respectively. In similar accordance the magnetization values presented a decrease in its values with milling time, from $\sim 127$ to $\sim 25 \mathrm{emu} / \mathrm{g}$. The dependence of both coercivity and 


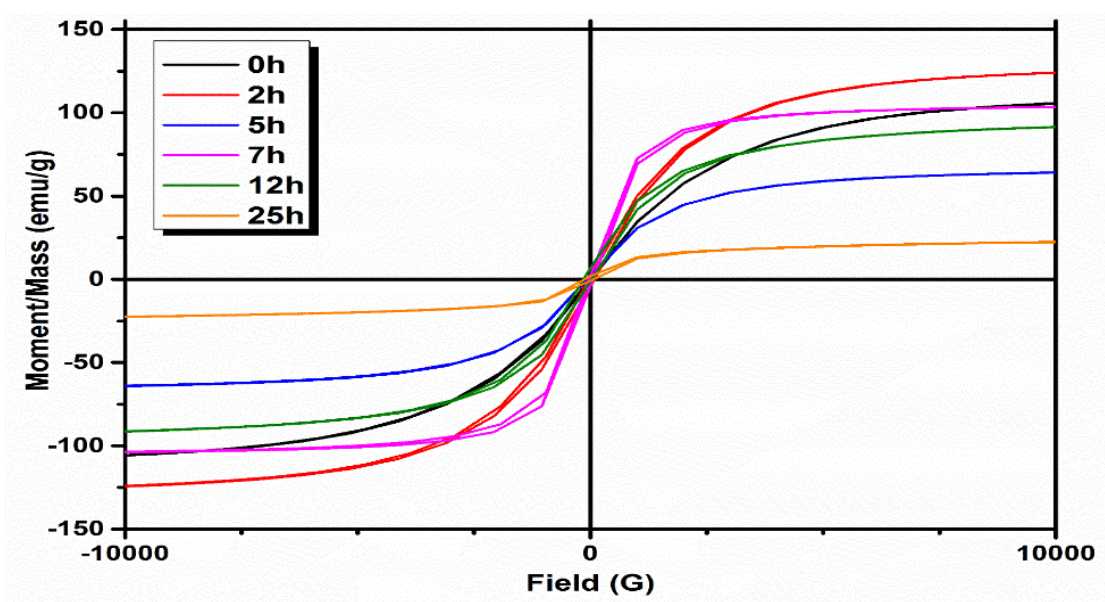

Fig. 4. Hysteresis curves of mechanically alloyed Fe-50wt.\% Cu powder with different times.
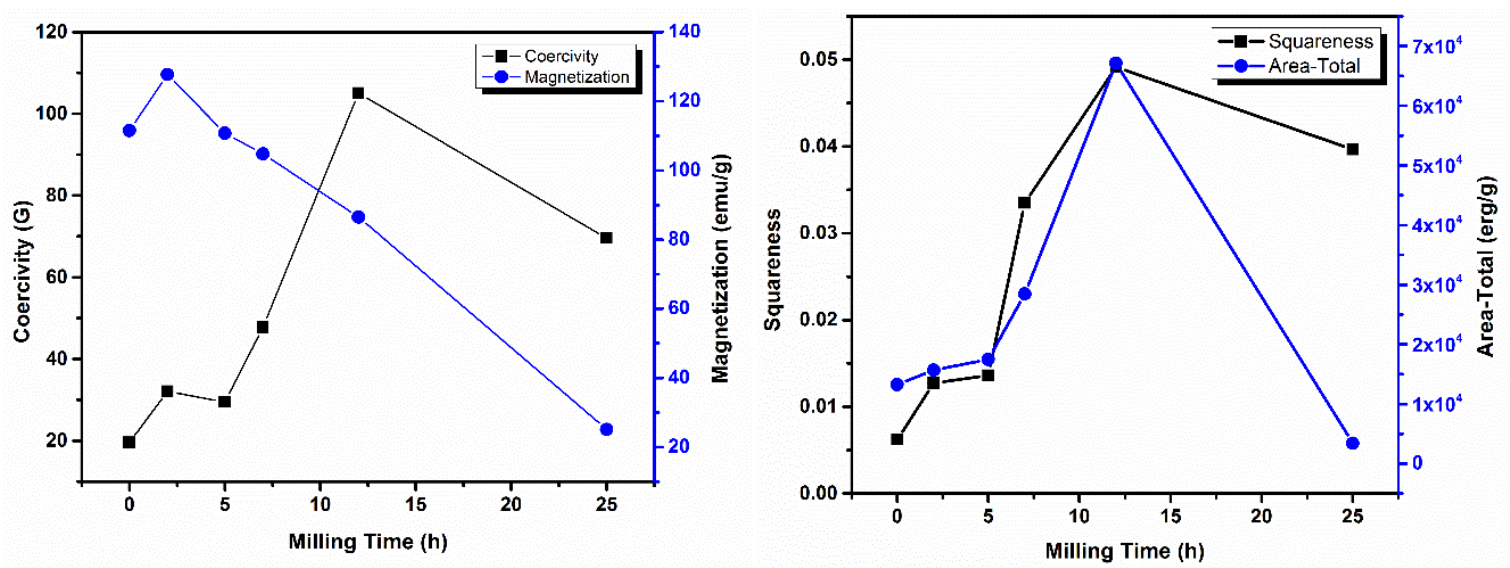

Fig. 5. Milling Time dependence of mechanically alloyed Fe-50wt.\% Cu powder; (a) Coercivity and magnetization and (b) Squareness and Area-Total.

magnetization of the Fe-50wt.\% Cu powder are in agreement with the XRD results, i. e. to the formation of a super-saturated $\mathrm{Cu}(\mathrm{Fe})$ limited solid solution phase.

It is reported that [14], an increase in the squareness, when going from the isolated nanocrystals to the $2 \mathrm{D}$ isotropic monolayer with the applied field in the surface. Then when we compare to the case of nanocrystals arranged in linear chains, the squareness is notably increased when the field is parallel to the chains. As shown in figure 5, the squareness of the hysteresis curve increases with milling time up to 12 hrs due to particle agglomeration and then decreases after particle refining.

At milling time higher than 12 hours up to 24 hours the coercivity begins gradual decreases and also the magnetization continue decreases in the same range of milling time but a different slope dependence. The measurements behavior of both coercivity and magnetization between 12 and $24 \mathrm{hrs}$ milling were in agreement with results suggested by XRD that postulated a further refinement and a mixture of an $\mathrm{Cu}(\mathrm{Fe})$ 
supersaturated phase was formed in addition to fcc $(\mathrm{Cu})$ which affected the values of coercivity and magnetization.

\section{CONCLUSION}

- Formation of supersaturated solid solution Fe-50wt.\%Cu during High-energy ball milling has been formed.

- A super-paramagnetic behavior was observed by VSM results of the as-milled samples with higher milling time corresponds to the fine structure formation (very small domain).

- The behavior of both the coericivity and the magnetization with milling time seem to be caused by the grain size reduction in agreement with hysteresis curves of mechanically alloyed sample with different milling times.

\section{REFERENCES}

[1] K. Venishi, K. F. Kobayashi, S. Nasu, H. Hatore, K. N. Ishibera, P. H. Shingu. Z. Mtallk 83, 132 (1992).

[2] A. R. Yavari, P. J. Desare, T. Benameur. Phys. Rev. Lett. 68, 2235 (1992).

[3] M. Moytahedi, M. Goodarzi, M. R. Aboutalebi, M. Ghaffari, V. Soleimanian, J. Alloy. Comp. 550, 380 (2013).

[4] J. Z. Jinng, C. Gente, R. Rormann. M. Sci. Eng. A 242, 268 (1998).

[5] P. Gorria, D. Martinez-Blanco, R. Iglesias, S. I. Palacios, M. J. Perez, J. A. Blanco,L. Fernandez Barquin, A. Hernando, M. A. Gonzalez. J. Mag. Magn. Mater. 300, 229 (2006).

[6] O. Drobhlav, A. R. Yavari. Acta metal. Mater. 43, 5, 1799 (1995).

[7] P. Crespo and J. A. Hernando, Recent Res. Level, Nanostructure, 1, 63 (1999).

[8] L. B. Hang and B. Fultz, Acta mater. 46, 2937 (1998).

[9] J. Z. Jiang, C. Gente, R. Bormann. Appl. Phys. Lett. 63, 2765 (1993).

[10] T. Li, Y. Z. Li, Y. H. Zhang, C. Gao, S. Q. Wei, W. H. Liu. Phys. Rev. B 52, 1120 (1995).

[11] E. Ma, H. W. Sheng, J. H. He and P. J. Schilling. Mate. Sci. Eng. A 286, 48 (2000).

[12] P. Bellon, R. S. Averbook. Phys. Rev. Lett. 74, 1819 (1995).

[13] C. Cente, M. Oehring, R. Bormann. Phys. Rev. B 48 (1993).

[14] V. Russier, C. Petit, and M. P. Pileni. J. Appl. Phys. 93, 12, 10001 (2003). 